\title{
11-Surfaces, Interfaces and Thin Films
}
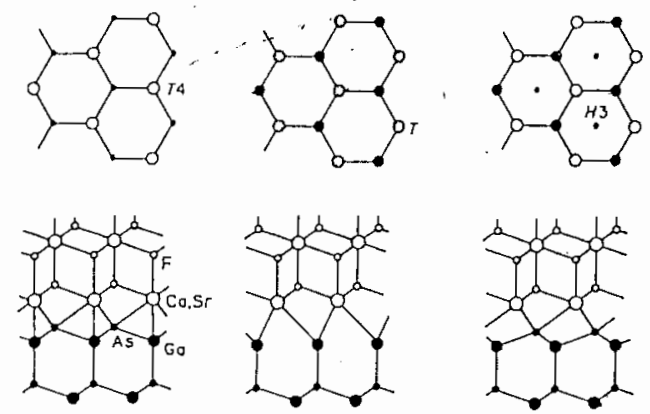

74

$T$ (miss.)

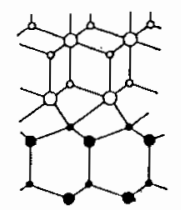

$T$ (shirt)

$\mathrm{CaF} 2 / \mathrm{S} / \mathrm{GaAs}(111)$ samples were prepared by growing 54 monolayers of $\mathrm{F}-\mathrm{Ca}-\mathrm{F}$ from a molecular-beam source on a sulfide-treated GaAs(111) surface, XSW data collected using $3.5 \mathrm{keV}$ photons support three-fold coordinated $\mathrm{S}$ atoms located in slightly shifted top-layer As atom sites. CaFz epilayers on the S-chemisorbed GaAs(111) surface have a good crystalline order in the vertical direction, but the first $F$ monolayer is again missing. The firstlayer $\mathrm{Ca}$ atoms are placed at a short vertical height close to 2.1 \& above the $S$ layer, which disfavors the on-top sites for the $\mathrm{Ca}$ atoms. The $\mathrm{CaF} / \mathrm{S} / \mathrm{GaAs}(111)$ interface was found to be more rough ( $\mathrm{rms}$ roughness $8 \sim 10 \AA$ ) than the $\mathrm{CaSrF} 2 / \mathrm{GaAs}(111)$ interface.

PS-11.02.23 PREPARATION OF C-ORIENTED POLYCRYSTALLINE ZnO THIN FILM BY SOL-GEL TECHNIQUE. By Zuoyan*Fangtian Gong, Jingyi Chang.Chima Building Material Academy, Beijing 100024, China. oriented polycrystalline $\mathrm{ZnO}$ thin films were prepared from metallo-organic compounds by a sol-gel method. The prearsor solution was synthesized with zinc acetate, acetylacetone, and alcohol through refluxing. Thin films were deposited on single-crystal $\mathrm{Si}(100), \mathrm{Si}(111)$ and fused silica using dip-coating technique. Coriented polycrystallime Zno thin films wewe obtained with a special isotnermal treatment. The pyrolysis and crystallization of powder and films were investigated by differential thermal analysis, thermogravimetric analysis, $x$-ray diffration and scanning electron microscope. The effects of substrate, sintering and other processing parameters on the crystal structure were also invest1gated. In this experiment, the crystal structure of trin films had a strong dependency on the neat treatment, while the substrate played a smaller role tnan we expectea.

Decomposition of gel films occured below $200^{\circ} \mathrm{C}$ Nucleation of films started at about $300^{\circ} \mathrm{C}$. The densified crystalline films were obtalned akove $500^{\circ} \mathrm{C}$, they transtormed to c-oriented fillms with increasing the temperature to $800^{\circ} \mathrm{C}$.
PS-11.02.24 A STUDY OF FE-DY MULTILAYERED FILMS. By Peixuan Wang, Shengli $\mathrm{Li}^{*}$ and Ruzhang $\mathrm{Ma}$, Department of Materials Physics, University of Science and Technology Beijing, Beijing 100083.

Fe/Dy compositionally modulated films were prepared by alternate evaporation of the two elements onto substrates in the vacuum of $10^{-7} \mathrm{~Pa}$ range. RBS and AES were used for the composition profile determinations, and XRD and TEM for microstructure observations. Measurements of magnetic properties were also performed with vibrating sample magnetometer. Two kinds of multilayers have been investigated. The first group has short periodic length, $\Lambda=4--$ $6 \mathrm{~nm}$ and constant chemical ratio Fe80Dy20. The second group has longer periodicity $\Lambda=25--50 \mathrm{~nm}$ and various compositions ranged between $\sim$ Fe90Dy 10 and $\sim$ Fe34Dy 66 .

TEM shows that the Dy layers are in the amorphous state when their thickness $<2.4 \mathrm{~nm}$, whereas Fe layers $>2.4 \mathrm{~nm}$ give typical diffraction patterns of bcc structure. These as-deposited structures are very unstable against the film heating. Sharp diffraction rings characteristic of hcp-Dy will take the place of diffuse rings of amorphous Dy in $\sim 60 \mathrm{sec}$ during irradiation with intense electron beam $(100 \mathrm{keV})$. Meanwhile the aggregation of $\mathrm{Fe}$ can also be observed. However, these processes occurred relatively slowly (in tens of minutes) when the films were heated at TEM hot stage.

For samples of the second group ion beam mixing have been studied with Ar ions. The ion energy of $95-110 \mathrm{keV}$ was selected so that the mean projected range of bombarding ions is about in the middle of the multilayers ( $100 \mathrm{~nm}$ thick). The amount of mixing, $Q$, of adjacent elemental layers can be determined from RBS spectra (Hewett C. A. et al., Nucl. Instr. Meth., 1985, B7/8, 597). It is found that $Q$ increases with increasing the ion fluence. For all the competitions studied, complete mixing (i. e. $Q=1$ ) can be achieved at $1 \times 10^{17}$ ions $/ \mathrm{cm}^{2}$. In particular, the samples of $\sim \mathrm{Fe} 60 \mathrm{Dy} 40$ exhibit a striking contrast to those of other compositions. For that , $\mathrm{Q}=0.8$ can already be obtained at $1 \times 10^{16} / \mathrm{cm}^{2}$. After irradiations with $1 \times 10^{17} / \mathrm{cm}^{2}$, these films consist of only amorphous compound as shown by XRD, while those of other compositions give extra reflections contributed either by excess bcc-Fe (e. g. in Fe80Dy20) or by excess hcp-Dy (e. g. in Fe34Dy66). As is expected, the saturation magnetization, Ms, of the as-deposited multilayers varies with respect to their Dy content. For all the films Ms drops sharply after bombardments with the least fluence used $\left(5 \times 10^{15} / \mathrm{cm}^{2}\right)$. Further increase in ion fluence up to $1 \times 10^{17}$ merely results in small change of Ms. Among all samples, those of $\sim$ Fe60Dy 40 show maximum reduction of $\mathrm{Ms}$ induced by ion beam mixing.

In this paper the behavior of multilayers depending on the composition is discussed in the connection with the phase diagram, the enthalpy of compounds formation and the magnetic coupling of Fe-Dy atoms. 